\title{
Pain here and now: physical pain impairs transcendence of psychological distance
}

\section{Jens Agerström \\ Kent Stening \\ Olof Axman}

Department of Psychology, Linnaeus University, 39/ 82 Kalmar, Sweden
Correspondence: Jens Agerström Department of Psychology, Linnaeus University, Pedalstråket II, 39231

Kalmar, Sweden

Tel +46480446068

Email jens.agerstrom@Inu.se
This article was published in the following Dove Medical Press journal: Journal of Pain Research

Background: The ability to traverse psychological distance by going beyond the experienced reality of the self, here and now, is fundamental for effective human functioning. Yet, little is known about how physical pain affects transcendence of psychological distance. Using a construal level theory framework of psychological distance, the current research examines the hypothesis that pain impairs people's ability to traverse any kind of psychological distance whether it be temporal, social, and spatial distance, or the hypothetical.

Methods: Using the cold pressor test, 151 participants participated in an experiment where they were either induced with acute pain (treatment group) or no pain (control group) while completing a battery of questions measuring to what extent their current thoughts were transcending psychological distance.

Results: The results were largely consistent with the hypothesis. Relative to the control group, pain induced participants showed significantly less transcendence of past temporal distance, social distance, spatial distance, and the hypothetical. Furthermore, greater self-reported pain intensity was significantly associated with less transcendence of temporal (past and future), social, and spatial distance.

Conclusion: Physical pain impairs the ability to traverse psychological distance. The research has practical implications for the pain clinic and for pain-afflicted individuals in everyday life. Keywords: physical pain, temporal distance, social distance, spatial distance, hypotheticality

\section{Introduction}

"Pain is the doorway to the here and now. Physical or emotional pain is an ultimate form of ground, saying, to each of us, in effect, there is no other place than this place, no other body than this body, no other limb or joint or pang or sharpness or heartbreak but this searing presence". ${ }^{1}$

The ability of traversing psychological distance by going beyond the experienced reality of the self, here and now, is fundamental for effective human functioning. ${ }^{2} \mathrm{We}$ need to be able to plan for the future, take other people's perspectives, envision remote places, and consider hypothetical outcomes. How is the ability to traverse psychological distance affected by physical pain? Is the poet David Whyte's above-cited claim that pain makes us focus on the here and now correct? The current research concerns this very question from a construal level theory (CLT) perspective ${ }^{3}$ examining the broader hypothesis that pain makes it more difficult to traverse any kind of psychological distance whether it be temporal distance, social distance, spatial distance, or hypothetical. Given the high prevalence of pain in the general population with millions of people being affected by daily pain and with $\sim 19 \%$ of adult Europeans suffering from moder- 
ate or severe chronic pain, ${ }^{4}$ its impact on fundamental human abilities are particularly important to study.

That pain narrows the attention to oneself, in the here and now, is consistent with Loewenstein's theory on the operations of visceral factors. ${ }^{5}$ The theory proposes that people who experience visceral factors (eg, drive states, moods, emotions) at high intensity levels pay little attention to visceral factors that have been experienced in the past, will be experienced in the future, and are experienced by other individuals. Such attentional narrowing is believed to be generally adaptive with visceral factors playing an important role in regulating behavior. Physical pain, for example, often signals the presence of a harmful factor in one's immediate (not geographically distal) environment. It further poses an actual somatic threat to the organism, which, unlike anxiety and worry, to a larger extent involve hypothetical danger and situations ("what-if" scenarios). Finally, physical pain should be attended to sooner rather than later in order to increase the probability of survival. ${ }^{5,6}$

In addition, the link between physical pain and constrained psychological distance is supported by neuroscientific evidence, showing that acute physical pain draws on activity in the prefrontal cortex that also plays an important role when people engage in prospection, retrospection, and social perspective taking. ${ }^{7,8}$ Not only do people use the same brain regions when they traverse various psychological distances, physical pain seems to compete for activity in these regions, presumably rendering the transcendence of psychological distance more difficult.

In addition to the arguments we have provided thus far to support the hypothesis that physical pain constrains psychological distance, it is important to note that all psychological distances are interrelated because they all share the same egocentric reference point in the self, in the here and now. ${ }^{2}$ Through repeated exposure to covariation of distances, people have formed cognitive associations that reflect positive correlations among distances. ${ }^{9}$ After all, the future is hypothetical whereas the present is real. Our friends tend to be more geographically close to us than socially distant people (eg, strangers), and so on. In our view, this interrelatedness would also add to a broad distance constraining effect of physical pain, incorporating all psychological distances. When a pain afflicted individual's attention is constrained on one distance dimension, this should produce a similar attentional constraint with respect to other distance dimensions. For example, when pain strikes and attention is directed toward the self (rather than others), the immediate (rather than the distant) future should also more cognitively salient due to existing cognitive associations among distances.

There are some previous research studies that have studied the effects of physical pain on outcomes related to psychological distance. One experimental study suggests that physical pain results in a weaker preference for delayed vs immediate monetary rewards. ${ }^{10}$ Another study finds that physical pain cause people to share less money with others in an Ultimatum Game. ${ }^{11}$ However, since both these studies involved monetary rewards, and since monetary rewards have been found to serve a pain relieving function, ${ }^{12}$ it is unclear if physical pain directly alters temporal and social perspectives when monetary rewards are not part of the distance manipulation.

More closely related to the current research, Hellström and Carlsson ${ }^{13}$ reported some interview data on the temporal orientation of people induced with pain in an experimental within subjects design. Although their primary research question was to examine if experimentally induced physical pain alters perceptions of temporal duration, the authors also asked their participants $(\mathrm{N}=15)$ if they had been thinking about the future. Consistent with the current hypothesis, all pain induced participants reported being entrapped in the present in one way or another, whereas this did not happen when they were in a pain-free state. Expanding on the Hellström and Carlsson study, the current research aims to provide a more formal analysis of temporal distance, using a much larger sample of research participants. More importantly, it examines the hitherto unexplored broader hypothesis that physical pain interferes with people's ability to traverse all kinds of psychological distance, as conceptualized by CLT, whether it being temporal, spatial, social, or hypothetical distance.

\section{Methods}

\section{Power analysis}

The statistical tool $\mathrm{G}^{*}$ Power was used to conduct an a priori power analysis, aiming for an experiment with $85 \%$ power to detect a moderate effect size corresponding to Cohen's $d$ of 0.5 . Setting a conventional significance level of $P<0.05$, two-tailed, a sample size of 73 participants would be required in each experimental condition $(\mathrm{N}=146)$.

\section{Participants and design}

One hundred and fifty-one students at a university in the southern part of Sweden participated in the experiment. Assignment to experimental condition was random (pain condition; $\mathrm{n}=78$; 38 males, 40 females; control condition; $\mathrm{n}=73$; 38 males, 35 females). Before participation, all par- 
ticipants signed an informed consent form. The study was conducted in accordance with the Declaration of Helsinki. It was approved by the regional ethics review board in Linköping (code: 2016/467-32).

\section{Pain induction method}

Pain was induced by a cold pressor apparatus (Nuve BM-302). The apparatus contained $10 \mathrm{~L}$ of circulating water, allowing the experimenter to set a constant water temperature anywhere between $0^{\circ} \mathrm{C}$ and $80^{\circ} \mathrm{C}$. The cold pressor task is an extensively used pain induction method in experimental research. In the current setup, the apparatus was programmed to maintain a constant water temperature of $5^{\circ} \mathrm{C}$ in the pain (treatment) condition. This would ensure a powerful pain manipulation while simultaneously ensuring that a large number of participants would follow through with the pain manipulation. ${ }^{14}$ In the no pain (control) condition, the water temperature was set to a neutral, non-painful temperature of $27^{\circ} \mathrm{C}$.

\section{Measures}

\section{Reported pain intensity}

A VAS was used to measure how much pain the participants experienced during the experimental session. The participants placed a mark on a $10 \mathrm{~cm}$ perpendicular line that had two endpoints: no pain and worst imaginable pain. The participants received a score $(0-10)$ based on the distance on the 10-cm line between the "no pain" anchor and the participants' mark. The VAS constituted the manipulation check. By correlating the pain-induced participants' pain ratings with the psychological distance measure, correlational evidence for the link between pain and distance could also be obtained.

\section{Psychological distances}

A total of 13 items were measured: temporal (4), spatial (3), social distance (3), and hypotheticality (3). In relation to each distance dimension, we asked participants to provide absolute ratings for proximity and distance, respectively, and relative ratings. As our hypothesis states that pain makes traversing distance more difficult, we only expect pain vs control participants to differ in their absolute ratings of distance and their relative ratings of distance vs proximity. It is moving beyond the reference point of the real self, here and now, that should become difficult when being in pain. Absolute ratings of psychological proximity were included to control for response bias, including the possibility that pain-induced participants would be prone toward providing extreme ratings across the entire measurement scale.

\section{Temporal dimension}

Participants indicated on a 5-point scale how well they could perceive the present, and how well they could imagine the past, and the future, respectively ( $1=$ not well at all, $5=$ very well). They also provided a future vs present relative rating, indicating to what extent their current thoughts were focused on the future relative to the present $(1=$ great focus on the present, $5=$ great focus on the future). Participants were not asked to provide a present vs past relative rating because it was assumed that most participants' current thoughts would be focused on either what was going on at the moment or what was going to happen next, and not on what has happened in the past.

\section{Social dimension}

Participants indicated on a 5-point scale how well they experienced their own thoughts and feelings, and how well they could imagine the perspective of other people ( $1=$ not well at all, $5=$ very well). They also provided a relative rating, indicating to what extent their current thoughts were focused on other people relative to oneself ( $1=$ great focus on myself, $5=$ great focus on other people).

\section{Spatial dimension}

Participants indicated on a 5-point scale, how well they could see things through their inside visual perspective, and how well they could see themselves from a spatially distant camera perspective $(1=$ not well at all, $5=$ very well $)$. They also provided a relative rating, indicating to what extent their current thoughts were focused on spatially distal relative to proximal objects $(1=$ great focus on proximal objects, $5=$ great focus on distal objects).

\section{Hypotheticality}

Participants indicated on a 5-point scale how well they experienced their real situation and how well they could imagine being in a different situation than the one they were actually experiencing ( $1=$ not well at all, $5=$ very well). They also provided a relative rating, indicating to what extent their current thoughts were focused on hypothetical relative to real events $(1=$ great focus on things that are actually happening, $5=$ great focus on things that might happen).

\section{Procedure}

University students enrolled in various education programs were invited to participate in a study on distraction and cognition that would involve completing a series of cognitive tasks while keeping one hand immersed in a water 
bath. They were informed that the water temperature could be perceived as painful/uncomfortable but that they could withdraw the hand from the water bath at any time if they felt the procedure would be too uncomfortable. In order to be able to participate in the study, all participants had to sign an informed consent form.

When seated in the laboratory, they were asked by the experimenter to immerse their left hand in the water bath up to processus styloideus. The 13 items pertaining to the different psychological distances were displayed on a computer screen. For each item, the participants reported their answer orally to the experimenter. Allowing sufficient time for the experimental manipulation to have an effect before participants began to complete the first item of the psychological distance scale, the experimenter ascertained that all participants had their left hand immersed in the water bath while he provided final instructions. The computer was programmed to present each proximity/distance item for 9 seconds. This time setting was chosen based on careful pilot testing as we wanted to ensure that our participants had sufficient time to complete each item. We further wanted to ensure that most participants would be able to complete all measures while maintaining their hand immersed in the cold water. Importantly, we ascertained that the time setting was the same for all participants because we were afraid that participants in the pain condition would otherwise hasten through the test session to terminate the pain induction as quickly as possible. In total, the psychological distance measure items were displayed for 117 seconds. Immediately after the pain manipulation was terminated, the participants completed the VAS.

A thorough debriefing concluded the session. As a compensation for having participated in the study, all participants were offered a lottery ticket worth approximately $\$ 3.41$. The participants were also given the opportunity to donate the value of the lottery ticket, selecting among different charitable organizations. This constituted a separate study examining whether choice of charity is jointly determined by previous pain exposure and specific characteristics of the charity.

The experiment was conducted between April and September 2017.

\section{Results}

\section{Manipulation check}

The VAS scores showed that the experimental pain manipulation was successful. The pain condition $(\mathrm{M}=6.92, \mathrm{SD}=1.78)$ reported experiencing substantially more pain than the con- trol condition $(\mathrm{M}=0.93, \mathrm{SD}=1.15)$. The mean VAS score of the pain condition fell within the moderate to severe pain range according to the cutoff values proposed by Boonstra et al. ${ }^{15}$

Eleven participants in the pain condition withdrew their hand from the water bath before they had completed all items of the psychological distance measures. Of these, ten participants nevertheless completed both the psychological distance and the VAS measures. Since these participants' pain intensity scores $(\mathrm{M}=8.67, \mathrm{SD}=1.32)$ on the 11 -point VAS revealed that they had experienced substantial pain that lingered throughout the entire test procedure, they were retained in the statistical analyses. The remaining participant did not complete either of the measures.

\section{Main results}

Independent sample $t$-tests were performed to evaluate the experimental effect of physical pain, examining differences between the pain-induced (treatment) group and the control group. To avoid too many pairwise comparisons, the absolute distance ratings and relative ratings were merged to form a transcendence of distance index within each psychological distance dimension. Mean scores were then calculated with larger mean scores indicating greater transcendence of distance. Our hypothesis states that pain makes traversing psychological distances more difficult. Thus, we only expected the distance indexes to be influenced by pain. As noted in the "Methods" section, the proximity ratings served as control ratings, and these were not predicted to be influenced by pain.

We used Bonferroni corrections to reduce the risk of type I error when performing pairwise comparisons (pain vs control group) on the different distance dimension indexes. When examining if there would be an effect of pain on the control/proximity ratings, no corrections were made. The reason for this was that we did not want to miss an effect of pain on these control measures that would work against our hypothesis. Viewed together, using a Bonferroni adjusted alpha level (0.01) to the five distance indexes, but not to the control/proximity ratings $(0.05)$, contributes to a more stringent test of our hypothesis.

The data followed normal distributions, allowing for parametric tests to be used. All reported analyses are twotailed. Missing data were not replaced.

\section{Transcending distance}

First, we performed independent sample $t$-tests to examine the experimental effect of pain on transcendence of distance 
Table I Effects of experimentally induced pain on transcendence of distance

\begin{tabular}{|l|l|l|l|l|l|l|l|l|}
\hline Distance & \multicolumn{2}{l}{ Pain group } & \multicolumn{2}{l}{ Control group } & t & df & P-value & Cohen's d \\
\cline { 2 - 8 } & M & SD & M & SD & & & & \\
\hline Past & 2.84 & 1.11 & 3.54 & 1.19 & 3.70 & 147 & $0.000 I^{*}$ & 0.61 \\
Future & 2.75 & 0.86 & 2.90 & 0.83 & 1.08 & 147 & 0.282 & 0.18 \\
Social & 2.07 & 0.74 & 2.80 & 0.78 & 5.90 & 146 & $0.000 I^{*}$ & 0.96 \\
Spatial & 2.29 & 0.89 & 2.71 & 0.67 & 3.11 & 130 & $0.002^{*}$ & 0.53 \\
Hypothetical & 2.16 & 0.87 & 2.57 & 0.82 & 2.96 & 142 & $0.004^{*}$ & 0.48 \\
\hline
\end{tabular}

Note: $* P$-value is significant when using a Bonferroni adjusted alpha level $P<0.01$.

Table 2 Correlations between self-reported pain intensity and transcendence of distance

\begin{tabular}{|l|l|l|l|l|l|l|}
\hline Measures & I & $\mathbf{2}$ & $\mathbf{3}$ & $\mathbf{4}$ & $\mathbf{5}$ & $\mathbf{6}$ \\
\hline I. Pain intensity & - & $-0.41 \mathrm{I}^{*}$ & $-0.310^{*}$ & $-0.246^{*}$ & $-0.368^{*}$ & -0.177 \\
2. Past & - & - & $0.372^{*}$ & $0.318^{*}$ & $0.483^{*}$ & $0.452^{*}$ \\
3. Future & - & - & - & $0.408^{*}$ & $0.366^{*}$ & $0.298^{*}$ \\
4. Social & - & - & - & - & $0.387^{*}$ & $0.500^{*}$ \\
5. Spatial & - & - & - & - & - & $0.413^{*}$ \\
6. Hypothetical & - & - & - & - & - & - \\
\hline
\end{tabular}

Note: *Denotes significant correlations; alpha level $P<0.05$.

(Table 1). Consistent with our hypothesis, the pain group showed significantly less transcendence of past temporal distance, social distance, spatial distance, and the hypothetical, when compared to the control group. The only exception was future temporal distance, which was unaffected by the pain manipulation.

Second, we correlated the pain-induced participants selfreported pain intensity ratings with transcendence of distance (Table 2). As expected, pain intensity was significantly associated with less transcendence of distance for all distance dimensions, the only exception being hypotheticality, where the correlation fell short of significance. It should also be noted that consistent with the CLT conceptualization that psychological distances are interrelated, all distances were positively correlated with one another.

\section{Proximity (control) ratings}

Next, we assessed if the pain manipulation influenced the proximity (control) ratings (Table 3 ). Speaking against a response bias explanation, the ratings pertaining to psychological proximity were not affected by the pain manipulation, the only exception being spatial proximity.

The correlations between self-reported pain intensity and these ratings painted a similar picture (Table 4). Pain intensity was not significantly associated with proximity except for spatial proximity, where greater intensity was associated with less proximity.
Table 3 Proximity (control) ratings as a function of physical pain

\begin{tabular}{|l|l|l|l|l|l|l|l|}
\hline \multirow{2}{*}{ Proximity } & \multicolumn{2}{|l|}{ Pain group } & \multicolumn{2}{l|}{ Control group } & \multirow{t}{*}{ df } & P-value \\
\cline { 2 - 5 } & $\mathbf{M}$ & SD & $\mathbf{M}$ & SD & & & \\
\hline Temporal & 3.90 & 0.85 & 3.75 & 0.88 & $\mathrm{I} .0 \mathrm{I}$ & $\mathrm{I} 48$ & 0.314 \\
Social & 3.64 & $1.1 \mathrm{I}$ & 3.79 & 0.99 & 0.92 & $\mathrm{I} 48$ & 0.359 \\
Spatial & 3.12 & 1.16 & $3.7 \mathrm{I}$ & 0.93 & 3.39 & $\mathrm{I} 45$ & $0.00 \mathrm{I}^{*}$ \\
Hypothetical & $4.0 \mathrm{I}$ & $\mathrm{I} .15$ & 4.16 & 0.80 & 0.93 & $\mathrm{I} 47$ & 0.354 \\
\hline
\end{tabular}

Note: $* P$-value is significant; alpha level $P<0.05$.

Table 4 Correlations between self-reported pain intensity and psychological proximity (control) ratings

\begin{tabular}{|l|l|l|l|l|l|}
\hline Measures & I & $\mathbf{2}$ & $\mathbf{3}$ & $\mathbf{4}$ & $\mathbf{5}$ \\
\hline I. Pain intensity & - & -0.023 & -0.008 & $-0.272^{*}$ & -0.025 \\
2. Temporal & - & - & $0.238^{*}$ & 0.161 & $0.289^{*}$ \\
3. Social & - & - & - & 0.147 & $0.440^{*}$ \\
4. Spatial & - & - & - & - & 0.227 \\
5. Hypothetical & - & - & - & - & - \\
\hline
\end{tabular}

Note: *Denotes significant correlations; alpha level $P<0.05$.

\section{Summary of results}

Taken together, our results support the hypothesis that physical pain makes traversing psychological distance more difficult. Pain-induced participants showed less transcendence of past temporal distance, social distance, spatial distance, and the hypothetical. Although future temporal distance was not influenced by the pain manipulation, self-reported pain intensity was significantly associated with less transcendence of future distance. As expected, pain intensity was also significantly associated with less transcendence of past temporal distance, social distance, and spatial distance. It was only transcendence of the hypothetical that deviated from this pattern, falling short of significance. More importantly, however, the experimental effect of pain on hypotheticality was significant.

As expected, the ratings pertaining to psychological proximity were generally not affected by the pain manipulation, nor did these ratings correlate with pain intensity, except for spatial proximity, an issue which we return to in the "Discussion" section. 


\section{Discussion}

The current research supports the hypothesis that pain renders transcendence psychological distance more difficult. It is the first to show that pain affects transcendence of a broad spectrum of psychological distances involving temporal distance, social distance, spatial distance, and hypotheticality. These finding have both theoretical and practical implications to which we now turn.

\section{Theoretical implications}

The current research extends CLT of psychological distance to the pain domain. ${ }^{3}$ The established causal link between pain and psychological distance contributes to an increased understanding of how physical pain shapes cognitive processes that are fundamentally intertwined with our everyday lives. CLT provides a unifying framework that can help us understand how a broad range of seemingly unrelated psychological phenomena involving time, space, sociality, and hypotheticality could be similarly affected by physical pain.

The results are also largely consistent with Loewenstein's theory on the operations of visceral factors, ${ }^{5}$ which proposes that people who experience visceral factors (eg, drive states, moods, emotions) at high intensity levels show attentional narrowing by paying little attention to visceral factors that have been experienced in the past, will be experienced in the future, and are experienced by other individuals. The current research contributes to the research literature on visceral factors by showing that the visceral factor of physical pain affects attentional narrowing in an even broader sense and in a more direct manner. Using the broader conceptualization of psychological distance, we directly measured the participants' ability to traverse numerous psychological distances in a relatively context-free manner, showing that acute pain makes it more difficult to traverse not only time and social distance but also spatial distance, and hypothetical. With regard to temporal distance, the current theoretical framework, unlike Loewenstein's, posits that the visceral factor of pain would not only cause people to show temporal myopia with respect to "goods" that are specifically related to pain, but rather a more general temporal myopia. That is, a person who experiences substantial pain should be less far-sighted in general, not only when it, for example, comes to choices between immediate vs delayed pain relief. Our finding that pain-induced participants reported greater difficulties traversing past temporal distance together with the statistically significant negative correlations between the pain intensity scores and traversing future temporal distance give some support for our reasoning that pain leads to a more general type of temporal myopia.

\section{Practical implications}

The finding that physical pain renders traversing psychological distance more difficult has numerous behavioral and practical implications. For example, compared to other patient categories, pain patients may be less inclined to commit to long-term relative to short-term treatment plans, posing a challenge for the clinician. They may also have a difficult time understanding the toll that their own misery takes on other family members as well, posing substantial challenges for everyday interpersonal relations. Furthermore, pain patients should find it difficult to see themselves being in an alternative (hypothetically pain-free) state, which may contribute to feelings of hopelessness and the development of depression. An egocentric focus on the self here and now likely poses significant challenges for clinicians and family members in their interactions with pain-afflicted individuals. Insofar as traditional pain management methods (eg, pain medication) successfully alleviate pain, they may help the patient to transcend distance. However, for patients who are not helped by such treatments, interventions may need to more directly target pain patients' ability to traverse psychological distance. Social perspective taking exercises performed together with family members could, for example, be one component of such an intervention program. We believe that our results extend beyond the clinic, however. At some point in their lives, most people experience an episode of substantial pain that should linger long enough for it to have potential consequences for behaviors that involve adopting a more distant perspective, such as self-control, ${ }^{16}$ prosocial behavior. ${ }^{17}$

\section{Caveats and future directions}

There were two aspects of our data that were unexpected. First, unlike past temporal distance, future temporal transcendence was not significantly affected by the experimental pain manipulation itself. However, the pain intensity ratings yielded correlational support for our hypothesis, as intensity was significantly associated with less transcendence of the future. Viewed together with the results from the Hellström and Carlsson study described in the introduction, the overall picture suggests that future temporal distance is also affected by pain. However, more research on this topic is clearly needed. ${ }^{13}$

Second, the experimental effect of pain on spatial proximity was significant, and pain intensity correlated with spatial proximity. Specifically, pain-induced participants reported that it was more difficult to adopt an inside visual perspective compared to control participants, and higher pain intensity was associated with greater difficulty adopting such 
a perspective. This was unexpected, and we speculate that it could be due to a poorly designed item that unintentionally introduced spatial distance rather than proximity. It is possible that when the participants were asked to adopt an inside view, they also focused on objects in the distance, and that is why the pain group performed worse than the control group. In retrospect, it would probably have been better to construct a proximity item that would be more compatible with the distance item (distant camera perspective of the self) in terms of how it was framed, by asking the participants to adopt a near camera perspective of the self instead. This way, only spatial distance would vary while the visual object (the self) would remain constant in both items.

We think that the aforementioned unexpected finding should be viewed in light of the robustness of the other results showing a significant, debilitating effect of pain on distance, but not proximity, including the transcendence of spatial distance index, which incorporated a relative item where pain-induced participants reported a less distal focus on a relative scale ranging from proximal to distal. That is, when a proximal and distal focus were pitted against one another, pain-induced participants reported a less distal focus.

The current study did not measure how distracting the pain experience was. The pain-induced participants who had their hand immersed in $5^{\circ} \mathrm{C}$ water likely experienced more distraction than the control participants who had their hand immersed in $27^{\circ} \mathrm{C}$ water. It is possible that distraction could contribute to the differences found between the pain and the control groups. We do not necessarily see distraction as a confound, but rather as an integral process through which pain exerts some of its effect on cognitive outcomes, see eg, Crombez et al. ${ }^{18}$ But for this to be the case, the cognitive functions in question need to be affected by distraction to begin with. More research focusing on the mediational role of distraction when studying the effects of pain on cognition is needed. Studies that could identify cognitive outcomes for which distraction plays, and does not play, a mediational role would add important insights to the current literature.

The current experiment induced acute experimental pain, and therefore, our results do not automatically generalize to people experiencing clinical pain. It could be argued, however, that compared to short-lived acute pain, persistent pain lasting for longer periods of time should have even greater potential to produce changes in cognitive styles, particularly when the pain is experienced at substantial intensity levels. Nevertheless, future research needs to examine if the ability to traverse psychological distance is impaired in clinical pain populations too. Researchers may also want to examine how certain pain qualities, such as its persistence and duration, contribute to such an impairment. Given that the findings of the current study replicate in clinical populations, another important task is to create and subsequently evaluate the effectiveness of interventions aimed to help pain patients expand their mental horizons in everyday life.

\section{Conclusion}

We started this paper by citing the poet David Whyte. Overall our findings support his claim that physical pain is the doorway to the here and now. It further suggests that the ability to traverse psychological distances involving time, space, sociality, and the hypotheticality is impaired when pain is experienced at substantial intensity levels. We believe that these findings are not only of theoretical importance because the ability to traverse distance is fundamental for effective human functioning in everyday life.

\section{Disclosure}

The authors report no conflicts of interest in this work.

\section{References}

1. Whyte D. [Poem] 2013 (December 12). Available from: https://www.facebook.com/PoetDavidWhyte/posts/painis-the-doorway-to-the-here-andnow-physical-or-emotional-pain-is-an-ultimate/1657797004246296/. Accessed September 1, 2018.

2. Liberman N, Trope Y. Traversing psychological distance. Trends Cogn Sci. 2014;18(7):364-369.

3. Trope Y, Liberman N. Construal-level theory of psychological distance. Psychol Rev. 2010;117(2):440-463.

4. Breivik H, Collett B, Ventafridda V, Cohen R, Gallacher D. Survey of chronic pain in Europe: prevalence, impact on daily life, and treatment. Eur J Pain. 2006;10(4):287-333.

5. Loewenstein G. Out of control: visceral influences on behavior. Organ Behav Hum Decis Process. 1996;65(3):272-292.

6. Crombez G, Eccleston C, De Vlieger P, Van Damme S, De Clercq A. Is it better to have controlled and lost than never to have controlled at all? An experimental investigation of control over pain. Pain. 2008;137(3):631-639.

7. Apkarian AV. A brain signature for acute pain. Trends Cogn Sci. 2013;17(7):309-310.

8. Buckner RL, Carroll DC. Self-projection and the brain. Trends Cogn Sci. 2007;11(2):49-57.

9. Fiedler K, Jung J, Wänke M, Alexopoulos T, et al. On the relations between distinct aspects of psychological distance: an ecological basis of construal-level theory. J Exp Soc Psychol. 2012;48(5): 1014-1021.

10. Koppel L, Andersson D, Morrison I, Posadzy K, Västfjäll D, Tinghög G. The effect of acute pain on risky and intertemporal choice. Exp Econ. 2017;20(4):878-893.

11. Mancini A, Betti V, Panasiti MS, Pavone EF, Aglioti SM. Suffering makes you egoist: acute pain increases acceptance rates and reduces fairness during a bilateral ultimatum game. PLoS One. 2011;6(10):e26008.

12. Becker S, Gandhi W, Elfassy NM, Schweinhardt P. The role of dopamine in the perceptual modulation of nociceptive stimuli by monetary wins or losses. Eur J Neurosci. 2013;191(Suppl 1):3080-3088.

13. Hellström C, Carlsson SG. Busy with pain: disorganization in subjective time in experimental pain. Eur J Pain. 1997;1(2):133-139. 
14. Agerström J, Gunnarsson H, Stening K. Does physical pain impair abstract thinking? J Cogn Psychol. 2017;29(6):748-754.

15. Boonstra AM, Schiphorst Preuper HR, Balk GA, Stewart RE. Cutoff points for mild, moderate, and severe pain on the visual analogue scale for pain in patients with chronic musculoskeletal pain. Pain. 2014;155(12):2545-2550.

16. Fujita K, Trope Y, Liberman N, Levin-Sagi M. Construal levels and self-control. J Pers Soc Psychol. 2006;90(3):351-367.
17. Agerström J, Björklund F. Moral concerns are greater for temporally distant events and are moderated by value strength. Soc Cogn. 2009;27(2):261-282.

18. Crombez G, Eccleston C, Baeyens F, Eelen P. The disruptive nature of pain: an experimental investigation. Behav Res Ther. 1996;34(11-12):911-918.

\section{Publish your work in this journal}

The Journal of Pain Research is an international, peer reviewed, open access, online journal that welcomes laboratory and clinical findings in the fields of pain research and the prevention and management of pain. Original research, reviews, symposium reports, hypothesis formation and commentaries are all considered for publication.
Dovepress

The manuscript management system is completely online and includes a very quick and fair peer-review system, which is all easy to use. Visit http://www.dovepress.com/testimonials.php to read real quotes from published authors. 